plex can bind stably to a single piece of DNA (i.e., one of the promoters upstream of a given $\mathrm{V}$ exon). This immediately gives rise to the punctate pattern of expression observed, as the limiting number of complexes leads directly to only a subset of $P c d h$ genes being active in each neuron. This type of mechanism would also lead to random monoallelic expression, with each neuron expressing the maternal allele, the paternal allele, both alleles or neither allele of each Pcdh gene (Fig. 1).

\section{Cell-specific identity}

The precise regulation of spatial and temporal expression of genes allows specification of different parts of the nervous system, just as it allows specification of different parts of a developing organism. Distinguishing very similar neurons can also be accomplished by the differential activities of various transcription factors. The stochastic monoallelic expression of $P c d h$ genes provides an additional layer of cellular specification to otherwise similar cells. The reason for the existence of different forms of $P c d h$ may lie in their collective ability to give distinct identities to adjacent cells that are otherwise transcriptionally identical. This would be a type of individual cell 'self versus nonself' distinction, potentially useful to neurons as they form specific synapses ${ }^{11}$.

Another notable example of neuronal self versus nonself distinction has emerged from recent single-cell analyses of the alterna- tive splicing of the Drosophila melanogaster Down syndrome cell adhesion molecule gene (Dscam), an immunoglobulin superfamily gene involved in specifying neuronal connections. The alternative splicing of Dscam is particularly notable, with 38,016 possible splice forms allowing 19,008 distinct extracellular forms and two alternative transmembrane domains: exon 4 has 12 possible forms, exon 6 has 48 possible forms, and exon 9 has 33 possible forms ${ }^{12}$. The variable exons are quite divergent so that the encoded immunoglobulin domains are predicted to have very different three-dimensional structures, and recent studies suggest that homophilic interactions are stronger than heterophilic interactions ${ }^{13}$.

Single-cell analyses of Dscam diversity led to the unanticipated finding that Dscam alternative splicing introduces a stochastic aspect to the generation of diversity of neurons ${ }^{14}$. Although different populations of neurons have few limitations on choice and are therefore probably not distinguished by Dscam, each individual neuron seems to be different from its neighboring cells by virtue of expressing different Dscam isoforms. This allows each neuron to know when a portion of its membrane is contacting itself as opposed to an adjacent neuron (that could be identical except for the Dscam difference). This self versus nonself awareness at the single-cell level allows two adjacent cells, with theoretically identical levels of transcription of every gene in the genome, to be distinct from each other ${ }^{11,14}$

It is noteworthy that the vertebrate Dscam genes studied to date do not show extensive alternative splicing. Similarly, although the $P c d h$ genes in vertebrates including fish all have similar genomic structures ${ }^{15}$, the homologs in Drosophila species have a simple organization. Thus, a new general principle emerging from a variety of systems suggests that going beyond the diversity of cell types afforded by normal modes of gene regulation is an important aspect of forming complex neural systems. In different systems, different genes are involved and the mechanisms are quite diverse. But in each case, a stochastic process leads to important differences among adjacent (and otherwise identical) neurons. As humans, we like to think of ourselves as individuals. It seems as though our neurons strive for individuality as well.

1. Singh, N. et al. Nat. Genet. 33, 339-341 (2003).

2. Esumi, S. et al. Nat. Genet. 37, 171-176 (2005).

3. Kohmura, N. et al. Neuron 20, 1137-1151 (1998).

4. Sugino, H. et al. Genomics 63, 75-87 (2000).

5. Wu, Q. \& Maniatis, T. Cel/ 97, 779-790 (1999).

6. Lewis, C.M. et al. Am. J. Hum. Genet. 73, 34-48 (2003).

7. Tasic, B. et al. Mol. Cell 10, 21-33 (2002).

8. Ngai, J. et al. Cell 72, 667-680 (1993).

9. Malnic, B. et al. Cel/ 96, 713-723 (1999).

10. Mombaerts, P. et al. Cel/ 87, 675-686 (1996).

11. Neves, G. \& Chess, A. Cold Spring Harb. Symp. Quant Biol. (in the press)

12. Schmucker, D. et al. Cell 101, 671-684 (2000).

13. Wotjowicz, W.M. et al. Cell 118, 619-633 (2004).

14. Neves, G. et al. Nat. Genet. 36, 240-246 (2004).

15. Tada, M.N. et al. Gene 340, 197-211 (2004).

\title{
Repeats hasten evolution?
}

We normally think of evolutionary change, such as that underlying differences between species of mammals, occurring over many tens of millions of years. Even 'rapid evolution', exemplified by the diversity that defines us as human individuals, occurs over tens or hundreds of thousands of years. But the extreme changes in dog morphology that have taken place over the last 150 years are exceptionally fast by normal evolutionary standards. What mechanism would allow for such accelerated change? One school of thought holds that adaptation occurs primarily through selection for single-base pair changes. Because these mutations tend to occur relatively infrequently, however, it seems unlikely that they could underlie the rapid changes in the dog. Another school of thought favors mutations in regulatory regions, leading to changes in levels and tissue specificity of gene expression, as means for rapid shifts in evolution.

Now, John Fondon and Harold Garner of the University of Texas at Dallas report evidence for selection acting on tandem repeats (Proc. Natl. Acad. Sci. USA 101, 18058-18063; 2004). Tandem repeats are plentiful, contributing to $3 \%$ of the human genome, and occur

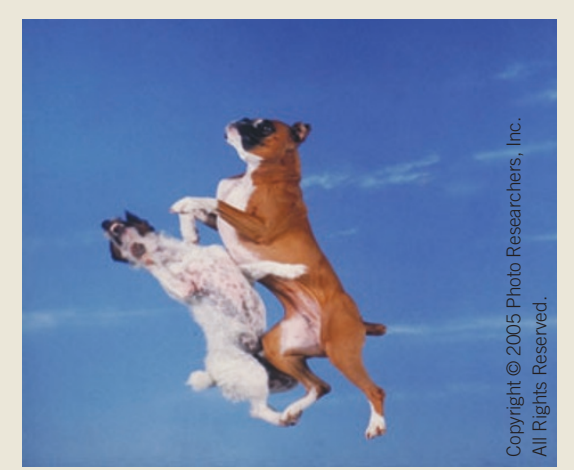
more frequently, $10^{5}$ times as often as single-point mutations. Selection acting on this class of mutations may, therefore, explain a faster rate of evolution. Fondon's study examined variation in tandem-repeat length in 142 dogs, including 92 domesticated breeds. The authors sequenced 37 repeat regions of 17 genes homologous to human and mouse genes involved in development and found significant variation in the number of repeats, correlated to several morphological phenotypes. This may not reflect typical evolution in the wild, particularly because domesticated dogs have undergone intensive artificial breeding selection for traits desirable to their owners, including morphological changes.

Most of the tandem repeats analyzed were located in coding regions. Although the function of these changes in repeat length remains to be explored, previous studies have shown that repeat expansions or contractions can cause either loss of function or hypomorphic alleles. In the human genome, expansion of triplet repeats has been associated with several hereditary neurological diseases, including fragile $X$ syndrome, Huntington disease, myotonic dystrophy and spinocerebellar ataxia. The function of repeat expansion or contraction in disease, as well as the relative prevalence and importance of tandem repeats compared with other types of mutations, are important questions to address in future studies in this emerging field.

Orli Bahcall 